\title{
When Reducing Low-Value Care in Hospital Medicine Saves Money, Who Benefits?
}

\author{
Joshua M. Liao, MD, MSc ${ }^{1,2,3 *}$, Amol S. Navathe, MD, PhD $3,4,5$, Marilyn M. Schapira, MD, MPH ${ }^{3,5,6}$, \\ Arlene Weissman, $\mathrm{PhD}^{7}$, Nandita Mitra, $\mathrm{PhD}^{3,8}$, David A. Asch, MD, MBA $3,4,5,6$
}

\begin{abstract}
${ }^{1}$ Department of Medicine, University of Washington, Seattle, Washington; ${ }^{2}$ Center for Scholarship in Patient Care Quality and Safety, University of Washington School of Medicine, University of Washington, Seattle, Washington; ${ }^{3}$ Leonard Davis Institute of Health Economics, University of Pennsylvania, Philadelphia, Pennsylvania; ${ }^{4}$ Department of Medical Ethics and Health Policy, University of Pennsylvania, Philadelphia, Pennsylvania; ${ }^{5}$ Center for Health Equity Research and Promotion, Philadelphia Veterans Affairs Medical Center, Philadelphia, Pennsylvania; ${ }^{6}$ Department of Medicine, University of Pennsylvania, Philadelphia, Pennsylvania; ${ }^{7}$ American College of Physicians, Philadelphia, Pennsylvania; ${ }^{8}$ Department of Biostatistics, Epidemiology and Informatics, University of Pennsylvania, Philadelphia, Pennsylvania.
\end{abstract}

One emerging policy solution for deterring low-value care is to financially penalize physicians who prescribe it. However, physicians' willingness to support such policies may depend on whether they perceive that benefits accrue to patients or to insurers and hospitals. We surveyed physicians practicing hospital medicine to evaluate the association between policy support and physician beliefs about who benefits from the money saved through reducing low-value services in hospital medicine. Overall, physicians believed that more of any money saved would go to profits and leadership salaries for insurance companies and hospitals and/or health systems rather than to patients. These beliefs were associated with policy support: $66 \%$ of those supporting physician penalties were more likely to believe that benefits accrue to patients or physicians, compared to $39 \%$ of those not supporting policies $(P<0.001)$. Our findings are consistent with a sense of healthcare justice, in which physicians are less likely to support penalties imposed on themselves if the resulting benefits accrue to corporate or organizational interests. Effective physician penalties will likely need to address the belief that insurers and provider organizations stand to gain more than patients when low-value care services are reduced. Journal of Hospital Medicine 2018;13:45-48. Published online first November 22, 2017 @ 2018 Society of Hospital Medicine hysicians face growing pressure to reduce their use of "low value" care-services that provide either little to no benefit, little benefit relative to cost, or outsized potential harm compared to benefit. One emerging policy solution for deterring such services is to financially penalize physicians who prescribe them. ${ }^{1,2}$

Physicians' willingness to support such policies may depend on who they believe benefits from reductions in low-value care. In previous studies of cancer screening, the more that primary care physicians felt that the money saved from cost-containment efforts went to insurance company profits rather than to patients, the less willing they were to use less expensive cancer screening approaches. ${ }^{3}$

Similarly, physicians may be more likely to support financial penalty policies if they perceive that the benefits from reducing low-value care accrue to patients (eg, lower out-of-pocket costs) rather than insurers or hospitals (eg, profits and salaries of their leaders). If present, such perceptions could inform incentive design. We explored the hypothesis that support of

\footnotetext{
*Address for correspondence and reprint requests: Joshua M. Liao, MD, MSc, UWMC Health Sciences, BB 1240, 1959 NE Pacific Street, Seattle, WA 98195;

Telephone: 206-616-6934; Fax: 206-616-1895; E-mail: joshliao@uw.edu
}

Received: April 25, 2017; Revised: July 12, 2017; Accepted: August 5, 2017

2018 Society of Hospital Medicine DOI 10.12788/jhm.2878 financial penalties for low-value care would be associated with where physicians thought the money goes.

\section{METHODS}

\section{Study Sample}

By using a panel of internists maintained by the American College of Physicians, we conducted a randomized, web-based survey among 484 physicians who were either internal medicine residents or internal medicine physicians practicing hospital medicine.

\section{Survey Instrument}

Respondents used a 5-point scale ("strongly disagree" to "strongly agree") to indicate their agreement with a policy that financially penalizes physicians for prescribing services that provide few benefits to patients. Respondents were asked to simultaneously consider the following hospital medicine services, deemed to be low value based on medical evidence and consensus guidelines ${ }^{4}$ : (1) placing, and leaving in, urinary catheters for urine output monitoring in noncritically ill patients, (2) ordering continuous telemetry monitoring for nonintensive care unit patients without a protocol governing continuation, and (3) prescribing stress ulcer prophylaxis for medical patients not at a high risk for gastrointestinal complications. Policy support was defined as "somewhat" or "strongly" agreeing with the policy. As part of another study of this physician cohort, 

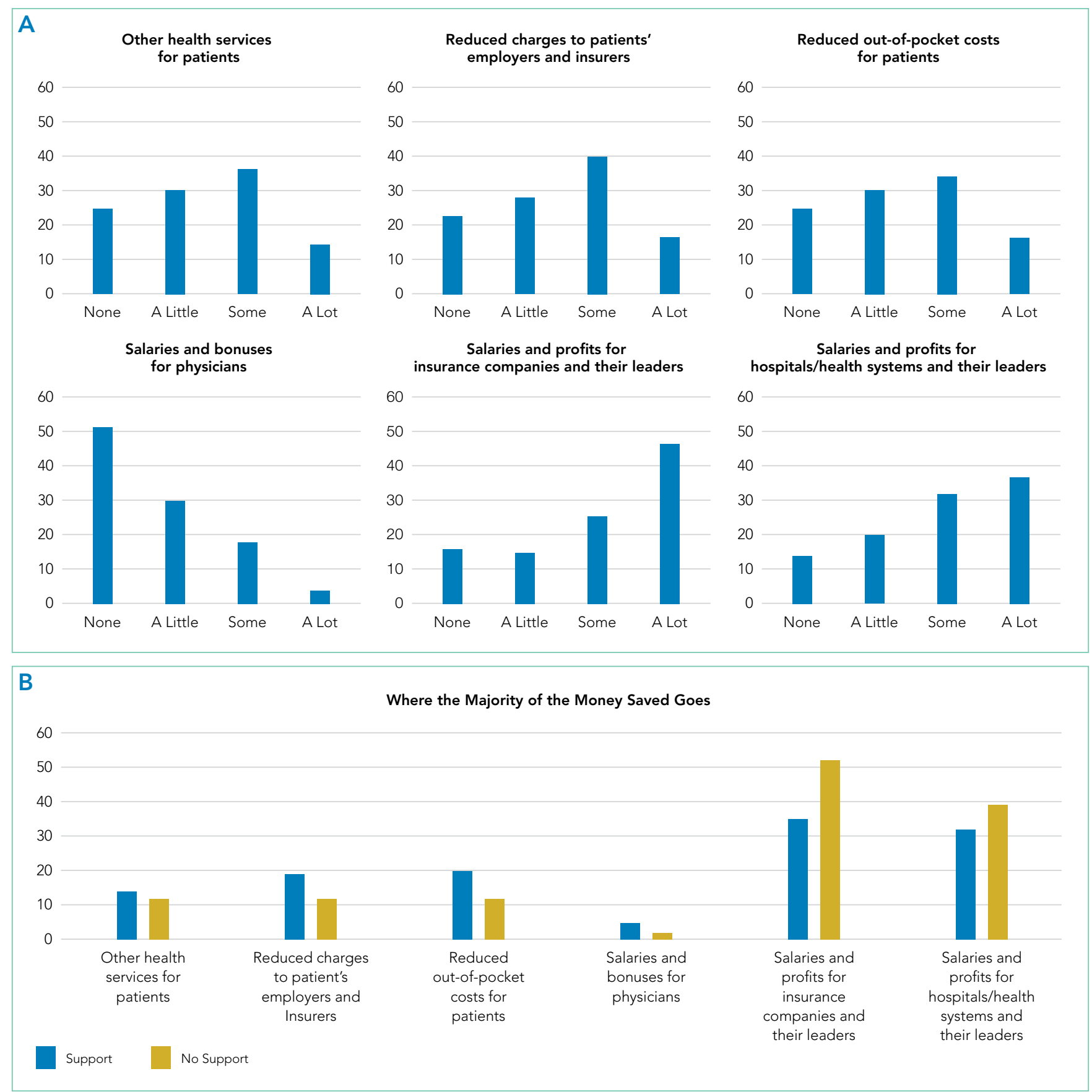

FIG. Physician Beliefs about where Money Saved from Reducing Low-Value Services Goes.

this question varied in how the harm of low-value services was framed: either as harm to patients, to society, or to hospitals and insurers as institutions. Respondent characteristics were balanced across survey versions, and for the current analysis, we pooled responses across all versions.

All other questions in the survey, described in detail elsewhere, ${ }^{5}$ were identical for all respondents. For this analysis, we focused on a question that asked physicians to assume that reducing these services saves money without harming the quality of care and to rate on a 4-point scale ("none" to "a lot") how much of the money saved would ultimately go to the following 6 nonmutually exclusive areas: (a) other healthcare services for patients, (b) reduced charges to patients' employers or insurers, (c) reduced out-of-pocket costs for patients, (d) salaries and bonuses for physicians, (e) salaries and profits for insurance companies and their leaders, and ( $f$ ) salaries and profits for hospitals and/or health systems and their leaders.

Based on the positive correlation identified between the first 4 items ( $a$ to $d$ ) and negative correlation with the other 2 items (e and $f$ ), we reverse-coded the latter 2 and summed all 6 into a single-outcome scale, effectively representing the degree to which the money saved from reducing low-value services 
TABLE. Odds Ratios for Physician Beliefs about Who Benefits from Reductions in Low-Value Care

\begin{tabular}{lcc}
\hline & \multicolumn{2}{c}{ Odds Ratios (95\% Cl) } \\
\cline { 2 - 3 } Variable & Unadjusted & Adjusted \\
\hline Policy Support & 1.00 & 1.00 \\
No & $3.1(1.7-5.7)$ & $2.8(1.5-5.3)$ \\
Yes & & $0.99(0.96-1.0)$ \\
\hline Age & & 1.00 \\
\hline Gender & & \\
Male & & $1.55(0.27-1.1)$ \\
Female & $1.1(0.39-2.9)$ \\
\hline Professional Status & \\
Practicing internist & \\
Resident & 1.00 \\
\hline Incentives & $1.2(0.57-2.6)$ \\
Cost & $0.63(0.30-1.3)$ \\
Noncost & $0.63(0.30-1.3)$ \\
None & & 1.00 \\
\hline Survey Version & & \\
Patient harm & & \\
Societal harm & & \\
\hline Institutional harm & & \\
\hline
\end{tabular}

NOTE: Abbreviation: $\mathrm{Cl}$, confidence interval.

accrues generally to patients or physicians instead of to hospitals, insurance companies, and their leaders. The Cronbach alpha for the scale was 0.74 , indicating acceptable reliability. Based on scale responses, we dichotomized respondents at the median into those who believe that the money saved from reducing low-value services would accrue as benefits to patients or physicians and those who believe benefits accrue to insurance companies or hospitals and/or health systems and their leaders. The protocol was exempted by the University of Pennsylvania Institutional Review Board.

\section{Statistical Analysis}

We used a $\chi^{2}$ test and multivariable logistic regression analysis to evaluate the association between policy support and physician beliefs about who benefits from reductions in low-value care. A $\chi^{2}$ test and a Kruskal-Wallis test were also used to evaluate the association between other respondent characteristics and beliefs about who benefits from reductions in low-value care. Analyses were performed by using Stata version 14.1 (StataCorp, College Station, TX). Tests of significance were 2-tailed at an alpha of .05 .

\section{RESULTS}

Compared with nonrespondents, the 187 physicians who responded (39\% response rate) were more likely to be female
(30\% vs $26 \%, P=0.001$ ), older (mean age 41 vs 36 years old, $P<0.001)$, and practicing clinicians rather than internal medicine residents $(87 \%$ vs $69 \%, P<0.001)$. Twenty-one percent reported that their personal compensation was tied to cost incentives.

Overall, respondents believed that more of any money saved from reducing low-value services would go to profits and leadership salaries for insurance companies and hospitals and/or health systems rather than to patients (panel A of Figure). Few respondents felt that the money saved would ultimately go toward physician compensation.

Physician beliefs about where the majority of any money saved goes were associated with policy support (panel B of Figure). Among those who did not support penalties, 52\% believed that the majority of any money saved would go to salaries and profits for insurance companies and their leaders, and $39 \%$ believed it would go to salaries and profits for hospitals and/or health systems and their leaders, compared to 35\% ( $P$ $=0.02)$ and $32 \%(P=0.37)$, respectively, among physicians who supported penalties.

Sixty-six percent of physicians who supported penalties believed that benefits from reducing low-value care accrue to patients or physicians, compared to $39 \%$ among those not supporting penalties $(P<0.001)$. In multivariable analyses, policy support was associated with the belief that the money saved from reducing low-value services would accrue as benefits to patients or physicians rather than as salaries and profits for insurance companies or hospitals and/or health systems and their leaders (Table). There were no statistically significant associations between respondent age, gender, or professional status and beliefs about who benefits from reductions in low-value care.

\section{DISCUSSION}

Despite ongoing efforts to highlight how reducing low-value care benefits patients, physicians in our sample did not believe that much of the money saved would benefit patients.

This result may reflect that while some care patterns are considered low value because they provide little benefit at a high cost, others yield potential harm, regardless of cost. For example, limiting stress ulcer prophylaxis largely aims to avoid clinical harm (eg, adverse drug effects and nosocomial infections). Limiting telemetric monitoring largely aims to reduce costly care that provides only limited benefit. Therefore, the nature of potential benefit to patients is very different-improved clinical outcomes in the former and potential cost savings in the latter. Future studies could separately assess physician attitudes about these 2 different definitions of low-value services.

Our study also demonstrates that the more physicians believe that much of any money saved goes to the profits and salaries of insurance companies, hospitals and/or health systems, and their leaders rather than to patients, the less likely they are to support policies financially penalizing physicians for prescribing low-value services.

Our study does not address why physicians have the beliefs that they have, but a likely explanation, at least in part, is that financial flows in healthcare are complex and tangled. Indeed, 
a clear understanding of who actually benefits is so hard to determine that these stated beliefs may really derive from views of power or justice rather than from some understanding of funds flow. Whether or not ideological attitudes underlie these expressed beliefs, policymakers and healthcare institutions might be advised to increase transparency about how cost savings are realized and whom they benefit.

Our analysis has limitations. Although it provides insight into where physicians believe relative amounts of money saved go with respect to 6 common options, the study did not include an exhaustive list of possibilities. The response rate also limits the representativeness of our results. Additionally, the study design prevents conclusions about causality; we cannot determine whether the belief that savings go to insurance companies and their executives is what reduces physicians' enthusiasm for penalties, whether the causal association is in the opposite direction, or whether the 2 factors are linked in another way.

Nonetheless, our findings are consistent with a sense of healthcare justice in which physicians support penalties imposed on themselves only if the resulting benefits accrue to patients rather than to corporate or organizational interests. Effective physician penalties will likely need to address the belief that insurers and provider organizations stand to gain more than patients when low-value care services are reduced.
Disclosure: Drs. Liao, Schapira, Mitra, and Weissman have no conflicts to disclose. Dr. Navathe serves as advisor to Navvis and Company, Navigant Inc., Lynx Medical, Indegene Inc., and Sutherland Global Services and receives an honorarium from Elsevier Press, none of which have relationship to this manuscript. Dr. Asch is a partner and partial owner of VAL Health, which has no relationship to this manuscript.

Funding: This work was supported by The Leonard Davis Institute of Health Economics at the University of Pennsylvania, which had no role in the study design, data collection, analysis, or interpretation of results.

\section{References}

1. Berwick DM. Avoiding overuse - the next quality frontier. Lancet. $2017 ; 390$ (10090):102-104.

2. Centers for Medicare and Medicaid Services. CMS response to Public Comments on Non-Recommended PSA-Based Screening Measure. https://www. cms.gov/Medicare/Quality-Initiatives-Patient-Assessment-Instruments/ MMS/Downloads/eCQM-Development-and-Maintenance-for-Eligible-Professionals_CMS_PSA_Response_Public-Comment.pdf. Accessed September 18, 2017.

3. Asch DA, Jepson C, Hershey JC, Baron J, Ubel PA. When Money is Saved by Reducing Healthcare Costs, Where Do US Primary Care Physicians Think the Money Goes? Am J Manag Care. 2003;9(6):438-442.

4. Society of Hospital Medicine. Choosing Wisely. https://www.hospitalmedicine.org/choosingwisely. Accessed September 18, 2017.

5. Liao JM, Navathe AS, Schapira MS, Weissman A, Mitra N, Asch DAA. Penalizing Physicians for Low Value Care in Hospital Medicine: A Randomized Survey. J Hosp Med. 2018;13(1):41-44. 